\section{NEUROGENESIS}

\section{RESTing repression}

Neurogenesis relies on a complex network of transcriptional activators and repressors that control the initiation and maintenance of neural traits. The molecular mechanisms involved in lineage commitments - from stem cells to terminally differentiated neurons - are not clear. Now, a study by Ballas and colleagues provides fascinating insight into how the progressive release of transcriptional repressors from promoters regulates the orderly expression of target genes during development.

In culture, embryonic stem (ES) cells can form embryoid bodies, which, when exposed to retinoic acid, differentiate first into neural progenitors and then into neurons. The authors found that the transcriptional repressor REST was expressed in abundance by ES cells, but was downregulated posttranslationally to minimal levels in neural progenitors. Interestingly, the residual REST still binds to the chromatin of neuronal genes, thereby repressing their expression, at the specific recognition site RE1 in neural progenitors; however, it is absent from the chromatin of the same genes in postmitotic neurons. This indicates that differentiation of progenitors into mature neurons requires the removal of REST-mediated transcriptional repression.

The silencing of neuronal genes by REST in differentiated non-neuronal cells involves a cofactor, CoREST, which recruits additional silencing machinery. This machinery includes specific histone methyltransferases, as well as methyl-CpG-binding protein (MECP2), which binds to DNA regions with a methylated cytosine-guanidine stretch ( $\mathrm{mCpG}$ ). In ES cells, a complex of REST, CoREST and MECP2 is present on the RE1 and $\mathrm{mCpG}$ sites in promoters of neuronal genes such as brain-derived neurotrophic factor $(B d n f)$ and Calbindin. The release of REST from the complex is sufficient for default expression of some neuronal genes; whereas other genes continue to be expressed at low levels owing to the presence of CoREST and MECP2 on the $\mathrm{mCpG}$ sites. The authors showed that membrane depolarization of differentiated neurons resulted in the release of MECP2 from the repressor complex on the $B d n f$ promoter and increased expression of the gene. Intriguingly, neuronal activity did not have the same effect on the repressor complex on the Calbindin promoter, which indicates that other factors might be necessary for the activation of Calbindin expression.

This elegant study indicates that specific transcriptional recognition sites and epigenetically-modified DNA sequences might provide a platform for the dynamic assembly and disassembly of repressor complexes that are required for lineage commitments and plasticity in mature neurons. How transcriptional activators might fit into the model remains to be seen.

(9) References and links

Jane Qiu

ORIGINAL RESEARCH PAPER Ballas, N. et al. REST and its corepressors mediate plasticity of neuronal gene chromatin throughout neurogenesis. Cell 121, 645-657 (2005) FURTHER READING Griffith, E. C. et al. REST acts through multiple deacetylase complexes. Neuron 31, 339-340 (2001) WEB SITE

Mandel's laboratory: http://www.hhmi.org/research/ investigators/mandel_bio.html

\title{
On the TRAIL of MS treatments
}

A protein that has well-described anti-inflammatory effects in animal models of multiple sclerosis (MS) might contribute to brain cell death in the disorder, according to the results of a new study. The finding offers the interesting possibility that suppressing the actions of TRAIL (tumour necrosis factor-related apoptosis-inducing ligand) in the CNS, while boosting its actions in the periphery, could improve clinical outcomes in MS

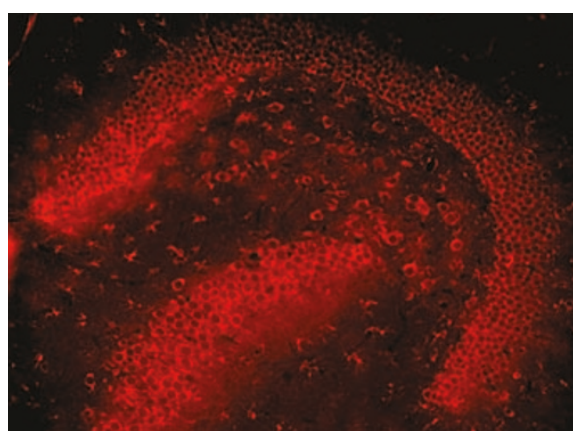

TRAIL receptor 2 immunofluorescence in the mouse hippocampus. Image courtesy of F. Zipp, Institute of Neuroimmunology, Humboldt University, Berlin, Germany.
Experimental autoimmune encephalomyelitis (EAE) has been used for decades to model immune events in the inflammatory, demyelinating disease MS. Previous studies have shown that TRAIL can inhibit the activity of autoreactive T cells - the mediators of pathogenesis in EAE - and that peripheral blockade of TRAIL can worsen EAE. What's more, TRAIL is upregulated in patients with MS who respond positively to interferon- $\beta$, an immunomodulatory treatment that is widely used in MS. But TRAIL is also known to induce apoptosis in human neurons and oligodendroglia in slice culture. Could this 'death ligand' have negative, neurodegenerative effects in EAE and MS?

To selectively block the actions of TRAIL in the brains of mice with EAE, Aktas and colleagues used TRAIL receptor 2 (TRAILR2 or DR5) fused to the antibody fragment Fc. Neuronal apoptosis in brainstem motor areas was markedly reduced in mice treated with the fusion protein, which was injected intracisternally after EAE had been triggered. Clinical disease scores also fell after DR5:Fc injection. The researchers went on to show that TRAILdeficient, myelin-specific T cells were less able to trigger EAE than were myelin-reactive cells from wild-type animals. Conversely, they found that the intracerebral delivery of TRAIL before or at the onset of EAE increased disease severity. Finally, TRAIL was shown to be a mediator of neuronal cell death induced by encephalitogenic $\mathrm{T}$ cells in organotypic brain slices.

By using EAE to model the neurodegenerative aspects of MS, Aktas and colleagues have shown that TRAIL contributes to neuronal apoptosis during autoimmune neuroinflammation. Could selectively inhibiting TRAIL in the CNS be of benefit to patients with MS? An important step in answering this question will be to determine whether the neuronal expression of TRAIL receptors is increased in the brains of patients. We await with interest further insights into the divergent functions of TRAIL.

Rebecca Craven

(2) References and links

ORIGINAL RESEARCH PAPER Aktas, O. et al. Neuronal damage in autoimmune neuroinflammation mediated by the death ligand TRAIL. Neuron 46, 421-432 (2005) FURTHER READING Lünemann, J. D. et al. Death ligand TRAIL induces no apoptosis but inhibits activation of human (auto)antigen-specific T cells. J. Immunol. 168, 4881-4888 (2002) | Wandinger, K. P. et al. TNF-related apoptosis inducing ligand (TRAIL) as a potential response marker for interferon- $\beta$ treatment in multiple sclerosis. Lancet 361, 2036-2043 (2003) 